\title{
Formulation Development and Characterization of Targeted Drug Delivery System for Breast Cancer
}

\author{
Rajkumari Thagele $^{* 1}$, Mohan Lal Kori ${ }^{2}$ \\ ${ }^{I}$ Professor, NRI Institute of Research \& Technology-Pharmacy, Bhopal, M.P., India \\ ${ }^{2}$ Principal, Vedica College of B.Pharmacy, RKDF University, Bhopal, M.P., India
}

\begin{abstract}
Cancer is one among the leading causes of death worldwide. Cancer cells proliferate at much faster rate than the normal cells. The available traditional cancer chemotherapy is not essentially selective as it depends on the kinetics of the cell growth. ${ }^{1}$ Breast cancer is common lethal cause of malignancy among women around all the countries. Early detection of breast cancer facilitates the diagnosis and treatment prior to metastasis. Despite remarkable development in new medicine findings and therapies for carcinoma during previous decades, no significant treatment methods are accessible for canceraffected people with invasive and metastatic carcinoma. During this stage, the patients have less response to cancer therapy thanks to recurrence properties of cancer.
\end{abstract}

Keywords - Wheat; Six Parameter Models; Gene Effects

\section{Introduction}

The incident of carcinoma is increasing in India and also this is often the second commonest cancer in rural Indian females [2]. Breast cancer detection at early stage has treatment like surgical resection with removal of axillary lymph nodes, radiotherapy, chemotherapy and hormone therapy [3]. Several risk factors for BC are well documented; however, for the bulk of girls with BC, it's impossible to spot specific risk factors. Nevertheless, some risk reduction could be achieved with prevention. WHO promotes $\mathrm{BC}$ control within the context of comprehensive national cancer control programmes that are integrated into non-communicable diseases and other related problems [4].

Recent years have seen significant effort devoted to formulate therapeutic agents in biocompatible nanocomposites such as nanoparticles, nanocapsules, micellar systems and conjugates as drug delivery systems. Application of nanotechnology for diagnosis, monitoring, disease therapy, and control of biological systems was referred to as "nanomedicine", and it has been receiving extensive attention over the past decade. Among these drug delivery systems, nanoparticles have received a considerable attention for the delivery of wide variety of drugs as well as biological macromolecules and vaccines. Nanotechnologies in general and nanoparticles in particular have revolutionalized the administration of medicines. Nanotecnology symbolize not only a miniaturization of larger objects but the preparation of nanomaterials with physical and chemical characteristics that differ from those of bulk materials, because they are on a nanometric scale. Depending upon the process used for the preparation of nanoparticles nanospheres (matrix type nanodevices) or nanaocapsules (reservoir type nanodevices) can be obtained [5].
Nanoparticles have been defined as submicron sized drug carriers, where the drug is either adsorbed on the surface or encapsulated within the particle. These nanoparticles can be prepared from natural and synthetic polymers that may or may not be biodegradable depending on their route of administration. the main advantages of nanoparticles is improved bioavailability by enhancing aqueous solubility, increasing resistance time within the body (increasing half-life for clearance/increasing specificity for its associated receptors and targeting drug to specific location within the body. This is why Nanoparticles are increasingly used in variety of applications that includes drug carrier systems and to pass organ barriers such as the blood-brain barrier, cell membrane, etc. The cellular uptake, biodistribution and circulating half-life are the key factors which are influenced by particle size of nanoparticles. Nanoparticles can also overcome the multiple drug resistance phenotype mediated by glycoprotein- $\mathrm{P}(\mathrm{P}-\mathrm{gP})$, resulting in increased drug content inside the cells and because of this reason biodegradable nanoparticles have been widely studied. Therefore, particle size becomes a primary concern while formulating a nanoparticulate system. Moreover the particle size thus obtained should be uniform because more uniform the distribution of particles more consistent will be the biodistribution, cellular uptake and drug release $[6,7]$. Oral administration of the non-steroidal anti estrogen like tamoxifen citrate is the treatment of choice for the patients with all stages of estrogen receptor (ER) - positive breast cancer. Antagonizing estrogen is popular treatment strategy because ER over expression is observed in about $70 \%$ of breast cancers, and about two-thirds of breast cancers in postmenopausal women are ER-positive. Oral tamoxifen citrate undergoes extensive hepatic metabolism and the subsequent biliary excretion of metabolites. Although the plasma antitumor concentration of 4-hydroxytamoxifen citrate are only about $2 \%$ of these of the parent compound 
this metabolite has been reported compound to be about 100 times quite as an tamoxifen than tamoxifen citrate. Tamoxifen citrate can have harmful long-term side effects such as the development of endometrial cancer, or an acquired tamoxifen citrate resistance leading to further tumor progression. Other side effects include liver cancer, increased blood clotting and ocular side effects such as retinopathy and corneal opacities [8] and development of drug resistance. These unwanted effects of tamoxifen citrate as well as various barriers to the effective administration of the drug to tumor demands targeted delivery to the site of tumor ad enhanced uptake by the tumor cells [9].

Recent research efforts have been directed towards developing safe and efficient chitosan based nanoparticulate drug delivery systems. Chitosan is a polysaccharide, composed of 2-amino-2-deoxy- $3-D$ glucan composed with glycosidic linkages. Compared to several other natural polymers, chitosan features a charge and is mucoadhesive. Therefore, it is used extensively in drug delivery applications. Chitosan is obtained from the deacetylation of chitin, a naturally occurring and abundantly available biocompatible polysaccharide chitosan is insoluble in acidic solution $(\mathrm{PH}<6.4)$ as a result of the protonation of the amino groups on the Dglucosamine residues. Because of its advantageous properties including biodegradability, biocompatibility, anti-bacteria and non toxicity, chitosan can be used in the fields of food processing, pharmaceuticals, cosmetics, biomaterials and agriculture [10]. In the present work, we focus on the preparation and systemic characterization of tamoxifen loaded biodegradable polymeric nanoparticles by the ionotropic gelation method. Effects of various process as well as formulation variables were carried out first to identify, in terms of small particle size and high encapsulation efficiency.

\section{Materials and methods Materials}

Tamoxifen citrate (Batch No.08160202 and TC/011/03/16) was received as gift from Khandelwal Laoratories Pvt. Ltd. Wagle Industrial Estate, Thane and Bioxera Pharma Pvt. Ltd. Ambernath (E), Thane, Maharashtra, India. Chitosan was obtained from Central Institute of Fisheries (Pune). All other chemicals were high purity grade and obtained from commercial source.

\subsection{Fourier Transform Infra Red Spectroscopy}

The infrared spectroscopy of the samples was carried out to ascertain identity of the drug and polymers. A mixture of was prepared by triturating $3-5 \mathrm{mg}$ of drug with $100-150 \mathrm{mg}$ of Potassium bromide in Quartz pestle and mortar. The mixture was then placed in sample holder in IR partition and scanned wave number $4000-450 \mathrm{~cm} \mathrm{-1}$ using FTIR spectrophotometer (Model IR Prestige- 21, Shimadzu, Japan). The observed peaks were compared with those reported (Brittain, 2011) for functional groups.

\subsection{Preparation of Tamoxifen Citrate Loaded Nanoparticles}

Chitosan nanoparticles were prepared as per procedure reported by Calvo et.al., (1997) with suitable modifications based on ionotropic gelation of chitosan with TPP anions. Chitosan $(2.0 \mathrm{mg} / \mathrm{ml})$ will be dissolved in aqueous acetic acid ( $\mathrm{pH} 4.0)$ and Sodium Tripolyphosphate $(1.0 \mathrm{mg} / \mathrm{ml})$ was dissolved in purified water. Drug (10\%) was added to TPP solution and stirred for 5 mins using magnetic stirrer.

Finally, $1.5 \mathrm{ml}$ of drug containing TPP solution was added to $4 \mathrm{ml}$ of the chitosan solution through syringe needle under magnetic stirring at room temperature thereby leading to formation of drug loaded chitosan nanoparticles at room temperature. The milky dispersion formed was centrifuged at $10000 \mathrm{rpm}$ at $4^{\circ} \mathrm{C}$ for $30 \mathrm{~min}$. The supernatant was discarded and sediments were sonicated for 30 mins and analyzed for further studies.

\subsection{Characterization of Nanoparticles}

\subsubsection{Particle Size Analysis}

The particle size, zeta potential and polydispersity index of prepared formulation was measured by Photon correlation spectrophotometer using Zeta Sizer Nano Series (Malvern Instruments Ltd., Malvern UK). The dispersion of NPs was diluted with purified water according to the mass concentration.

\subsubsection{Transmission Electron Microscopy}

TEM analysis was performed to examine the morphological characteristics of the formulation. The samples were stained with $2 \%$ (w/v) phosphotungustic acid for $30 \mathrm{~s}$ and placed on copper grids with films for viewing at an accelerating voltage of $200 \mathrm{kV}$.

\subsubsection{Zeta Potential Analysis}

Zeta Potential Analysis was measured using the Zetasizer NanoSeries (Malvern Instruments Ltd., Malvern UK). The sample diluted with distilled water. The $\mathrm{pH}$ was between 6.0and 7.0.

\subsubsection{Entrapment Efficiency}

For determination of entrapment efficiency of formulation, methanol was used as a solvent and determined spectrophotometrically at $276 \mathrm{~nm}$ against 
solvent blank. Entrapment efficiency was calculated by given formula.

Entrapment Efficiency $(\%)=\frac{\text { Amount of Drug in Nanoparticles }}{\text { Amount of drug added }} \times 100$

\section{Results and Discussion}

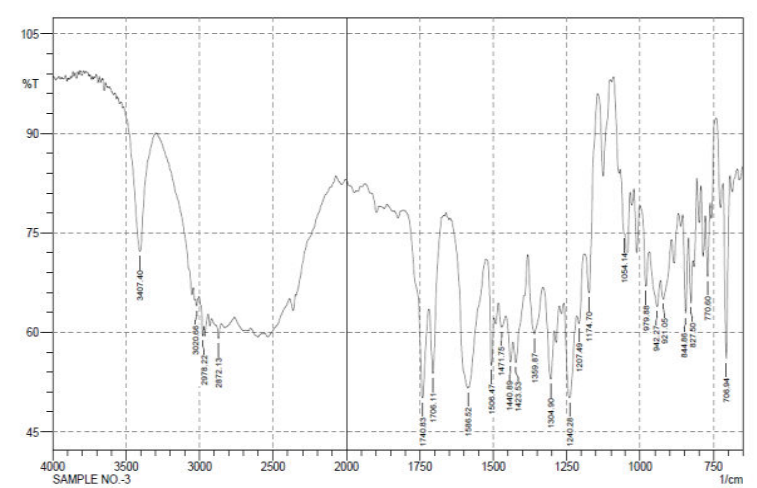

Fig.1: Fourier Transform Infra Red Spectroscopy

From FTIR of tamoxifen citrate pure drug (Fig No. 3) we could interpret major 3 peaks of functional groups $\mathrm{C}=\mathrm{C}$ - stretching $\mathrm{C}-\mathrm{H}$ stretching and $-\mathrm{NH}_{2}$. Their Freqency is 1507 and $1444, \mathrm{~cm}^{-1} 3027 \mathrm{~cm}^{-1}, 3200-3500 \mathrm{~cm}^{-1}$ respectively.

\subsection{Formulation of Tamoxifen Citrate Loaded Nanoparticles}

In our study, nanoparticles were prepared by high speed magnetic stirrer and ultrasound method that does not involve large amounts of excipients. The molecular interactions of the ionic cross linking of chitosan with sodium tripolyphosphate have been investigated. The studies have shown that process parameters have a significant effect on the particle size of the nanoparticles systems. It has been shown that there's a relationship between the high speed magnetic stirring and K.E. of the system. This K.E. causes the system to load static energy causing an agglomeration of the tiny particles. To avoid this, Ultra sonication plays significant role in obtaining a formulation characterized by unimodal narrow allocation because without ultarsonication, Polydispersity index of formulation was too high.

\subsection{Particle size Analysis}

Photon correlation spectroscopy is a technique to determine the mean particle size and the width of particle size distribution expressed as Polydispersity Index. The measurement using PCS is based on the light scattering phenomena in which the light intensity fluctuations of the scattered light from the particles in the measuring cell are measured. Here particle size recorded as Intensity. PDI index shows the particle size distribution for small colloidal nanoparticle dispersion. The size of prepared NPs was found to be $1629 \mu \mathrm{m}$.

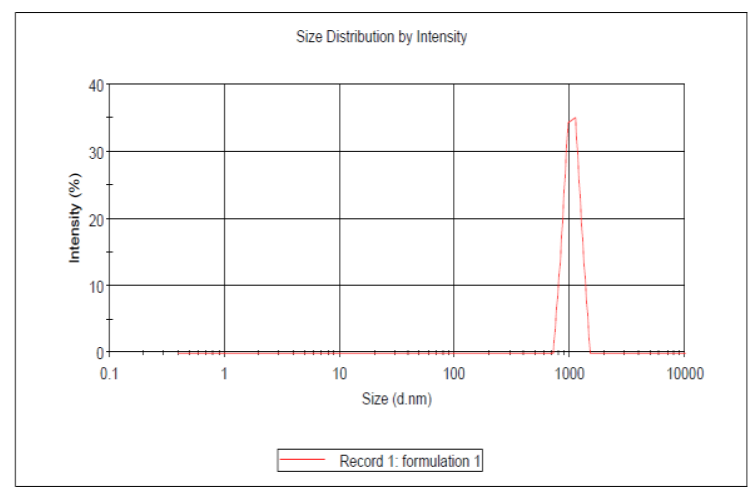

Fig. 2: Particle size

\section{Conclusions}

The results obtained in this work suggest that NPs have a great potential for drug delivery future prospects. They offer a great way of delivering drugs at a higher efficacy and lower toxicity.

\section{References}

[1] Jagadeesh G Hiremath, V Kusum Devi (2011). Preparation and in vitro characterization of poly(epsilon-caprolactone)-based tamoxifen citrate-loaded cylindrical subdermal implant for breast cancer. Asian journal of pharmaceutical sciences: 9-14.

[2] Jun H.Lee and Anjan Nan (2012). Combination of drug delivery approaches in metastatic Breast Cancer. Jou. of Drug Delivery. 1-17.

[3] Mali Deeapak, Nanjwade Basavaraj, Manvi F.V. (2013). Formulation and charactertization of Tamoxifen loaded stealth lipososmes for breast cancer. International Journal of Drug Development and Research. 5 (1):271-278.

[4] Naif Abdullah Al-Dhabi, (2016) Srisesharam Srigopalram,Soundharrajan Ilavenil,Young Ock Kim, Paul Agastian, Rajasekhar Baaru, Kannan Balamurugan, Ki Choon Choi, and Mariadhas Valan Arasu Proteomic Analysis of Stage-II Breast Cancer from Formalin-Fixed Paraffin-Embedded Tissues. BioMed Research International. Volume.1-6.

[5] Navneet Sharma, Parshotam Madan, Senshang Lin (2016) Effect of process and formulation variables on the preparation of parenteral paclitaxel-loaded biodegradable polymeric nanoparticles : A cosurfactant study. Asian L. of pharmaceutical sciences 11: 404-416.

[6] Özgür Erdem1 and Ezzettin Tokta G .Knowledge, Attitudes, and Behaviors about Breast Self-Examination and Mammography among Female Primary Healthcare Workers in DiyarbakJr, Turkey. BioMed Research International.1-6.

[7] P. Convreur (2013). Nanoparticles in drug delivery: Past, Present and Future.Advanced Drug Delivery Reviews.65: 21-23.

[8] Prajna Mishra, Bismita Nayak, R.K. Dey (2016). PEGylation in anticancer therapy: An overview. Asian journal of pharmaceutical sciences 11: 337-348.

[9] Rajath Othayoth1 K. Santhosh Kumar2 \& Varshini Karthik (2013). Development and Characterization of Chitosan-Pluronic Polymeric Nanoparticles for the Breast Cancer Treatment. International Journal on Mechanical Engineering and Robotics. Volume-1(1):71-79.

[10] Sanjeev R. Acharya a, Padmanabha R.V. Reddy (2016). Brain targeted delivery of paclitaxel using endogenous ligand. Asian journal of pharmaceutical sciences 11:427-438. 